\title{
Influences of the Tibetan plateau on tidal gravity detected by using SGs at Lhasa, Lijiang and Wuhan Stations in China
}

\author{
Heping Sun ${ }^{1,2}$, Huikang Zhang ${ }^{1,2}$, Jianqiao $\mathrm{Xu}^{1, *}$, Xiaodong Chen $^{1}$, Jiangcun Zhou ${ }^{1}$, and Miaomiao Zhang ${ }^{1}$ \\ ${ }^{1}$ Institute of Geodesy and Geophysics, Chinese Academy of Sciences, Wuhan, China \\ ${ }^{2}$ University of Chinese Academy of Sciences, Beijing, China
}

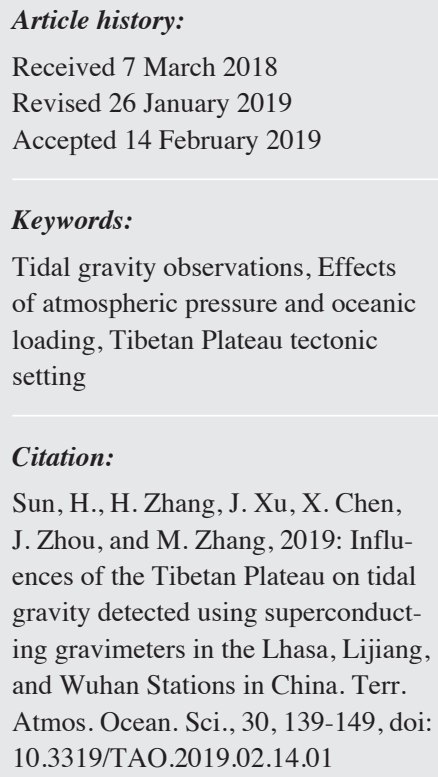

\begin{abstract}
We accurately retrieved tidal gravimetric parameters using long-term continuous tidal gravity measurements recorded by superconducting gravimeters (SGs) at stations in Lhasa, Lijiang, and Wuhan, China. We used these results to investigate the influences of the special tectonic setting on regional tidal deformation. We accurately evaluated scale factors of SGs with high precision better than $0.06 \%$. We carefully removed the effects of barometric pressure and oceanic loading from tidal gravity observations. We did not observe any obvious differences in amplitude factors for main tidal gravity waves for the stations in Lhasa and Lijiang. In the plateau area, we found the amplitude factor to be about $0.34 \%$ larger than that in the plain (Wuhan). Our study shows for the first time that the main reason for such tidal gravity anomalies can be explained by the influence of the special tectonic setting in the Tibetan Plateau.
\end{abstract}

\section{INTRODUCTION}

Gravitational tides are the resultant periodic changes in gravity fields associated with the Earth's deformation under tidal-generating forces from celestial bodies, including the Sun, the Moon, and the other planets. These tidal-generating forces are the dominant components in temporal gravity variation, and the largest magnitude of these forces is more than $250 \mu \mathrm{Gal}$ on the surface of the Earth. Previous research has shown that the tidal gravity change consists mainly of discrete harmonic signals located in long-period, diurnal (D), semidiurnal (SD), and terdiurnal (TD) frequency bands (Melchior 1994). The characteristics of gravity tides usually are described by using gravimetric parameters of the individual tidal waves, including the dimensionless amplitude factor $\delta$ and phase difference $\Delta \phi$. The amplitude factor is defined as the ratio of observed tidal gravity changes to those of a rigid Earth under tide-generating forces at the

\footnotetext{
* Corresponding author

E-mail: heping@asch.whigg.ac.cn
}

same location on Earth's surface, and is a linear combination of Love numbers. The research also shows that the gravimetric parameters, as well as the associated Love numbers, are comprehensive reflections of the Earth's interior structure and dynamical processes, including the layered spheres, the physical properties of the medium, Earth's rotation, free oscillation, free core nutation and so on. Theoretically, the gravimetric parameters can be modeled by means of a numerical integral of the deformation equations of the Earth's tides (Wahr 1981; Dehant et al. 1999; Mathews 2001; Xu and Sun 2003; Métivier and Conrad 2008). On the basis of accurate astronomical ephemerides deduced from modern astrometry, tide-generating potential (forces) can be modeled accurately at any place on the Earth's surface (Doodson 1921; Hartmann and Wenzel 1995; Roosbeek 1996). Meanwhile, these gravimetric parameters also can be retrieved accurately from long-term continuous tidal gravity measurements (Melchior et al. 1996; Xu et al. 2004; Sun et al. 2005b; Hinderer et al. 2015). Therefore, precise tidal 
gravity observations can reveal the Earth's exact response to these tide-generating forces and can contain abundant information about the structure of Earth's interior.

The Tibetan Plateau is located in the convergent region of the Pacific, Indian, and Eurasian plates. It is the largest and highest plateau in the world and also has the youngest orogeny. Recent measurements from global positioning system in the plateau and its adjacent regions have suggested that the Tibetan Plateau is undergoing extremely inhomogeneous strong deformation. This deformation shows that the crust is shrinking in a north-south direction and stretching in an east-west direction as a result of the continuous subduction and collision of the Indian plate into the Eurasian plate (Wang et al. 2001; Shen et al. 2005; Liang et al. 2013). Seismic and gravity studies have suggested that the distribution of crust thickness is extremely complicated in this region, at about $70 \mathrm{~km}$ on average and nearly two times the mean crust thickness of the Earth (Zhang et al. 2011; Shin et al. 2015; $\mathrm{Xu}$ et al. 2017). Additionally, the lithosphere of the Tibetan Plateau has strong heterogeneity and anisotropy (Yao et al. 2010). This raises two questions: Can this special tectonic unit significantly influence the regional tidal response? And if so, how large is that influence?

Due to the advantages of very high sensitivity and stability, extremely low noise level and drift, and broad frequency range of dynamic linear responses, the superconducting gravimeters (SGs) play an important role in study of geophysics and geodynamics (Melchior et al. 1996; Sun et al. 1999, 2003; Xu and Sun 2003; Hinderer and Crossley 2004; Crossley and Hinderer 2009).
During the past decades, the Institute of Geodesy and Geophysics, Chinese Academy of Sciences, successively established three permanent SGs at stations in Wuhan, Lhasa, and Lijiang (see the locations in Fig. 1). Lhasa is located in the southern part of the Tibetan Plateau near the northern side of the Himalayan Mountains. It is situated on flat land in a valley near the middle reaches of the Lhasa River, a tributary of the Brahmaputra River, with a crust thickness of about $75 \mathrm{~km}$. Lijiang is located near the eastern-southern boundary of the Tibetan Plateau, and Wuhan is located in the central area of the ancient stable Yangzi platform, far from the plateau, with a crust thickness of about $34 \mathrm{~km}$. All three SGs are the same type of model manufactured by GWR Instruments (San Diego, CA, USA) and include recording system. The latitudes for these three stations are more or less the same, such that the effects of ellipticity and rotation of the Earth on tidal gravity observations are nearly equal, and can be negligible in our case. Because the Tibetan Plateau is far away from the sea regions, the loading effects of the oceanic tides at the stations in Lhasa and Lijiang are relatively weak compared with station in Wuhan.

Researchers have attempted to make associations between the Earth's tidal gravity response and the physical properties of the lithosphere for years. Using global distribution observations from 227 tidal gravity stations and the regression technique, Shukowsky and Mantovani (1999) obtained an association between tidal gravity response and average blocks with the characteristic size of homogeneous lithosphere segments. They also showed a high correlation between $\mathrm{M}_{2}$ tidal gravity anomalies and lithosphere effective

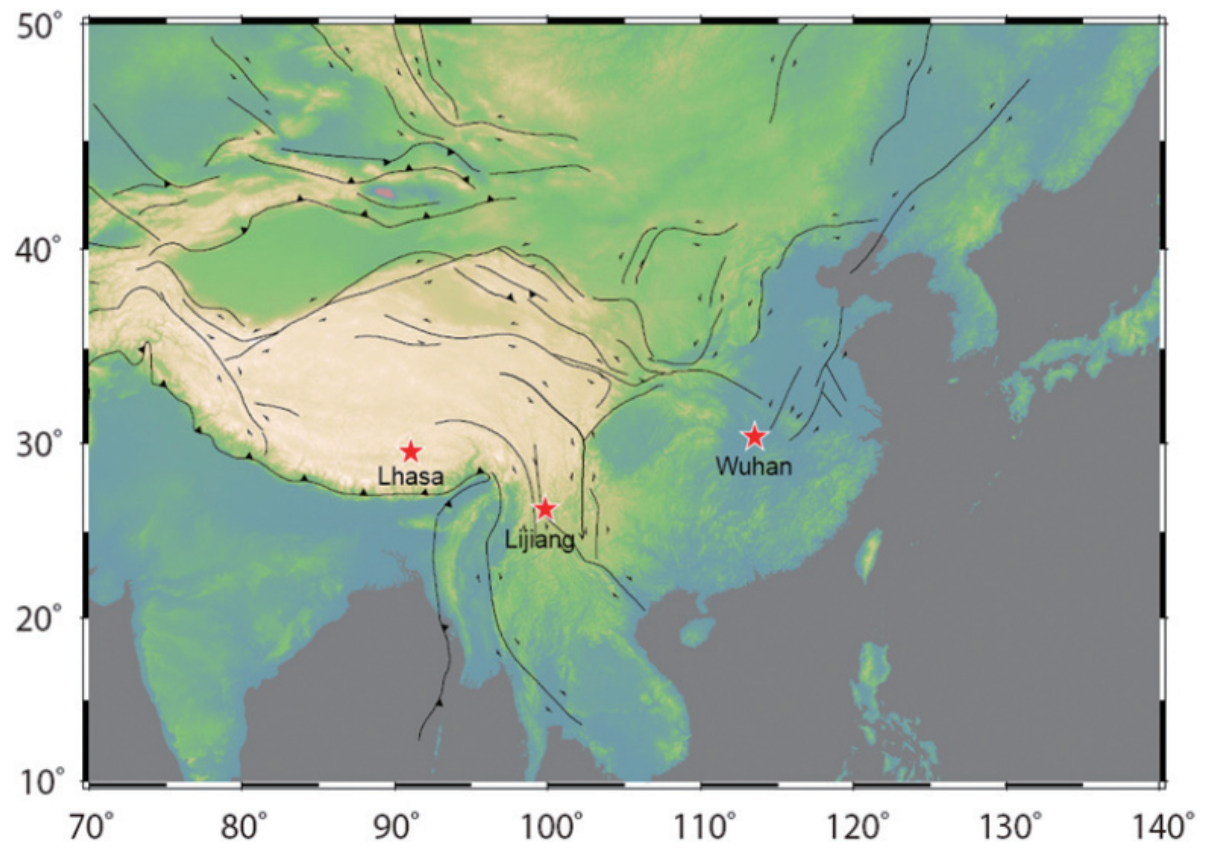

Fig. 1. Locations of the SG stations. 
elastic thickness. Latychev et al. (2009) used a finite volume numerical scheme to predict the response of a set of 3-D Earth models to a tide-generating potential while considering all three tidal bands. When they performed a series of numerical tests while also considering the elastic moduli, density, and dynamic topography, the resultant surface gravity changed in the semi-diurnal band by about $0.2 \mu \mathrm{gal}$.

The purpose of this study is to gain knowledge about the local influences of the Tibetan Plateau on regional tidal response. We used the SGs at three stations in Wuhan, Lhasa, and Lijiang to make continuous tidal gravity observations. We retrieved the gravimetric parameters from two years of continuous SG measurements, for which we carefully considered the loading effects of global and regional oceanic tides and local barometric pressure.

\section{PREPARATION OF THE SG OBSERVATIONS}

In this study, we select two years of continuous recordings in Lhasa from 1 January 2011, to 31 December 2012, and in Lijiang and Wuhan from 1 January 2015 to 31 December 2016. Because of a broken SG data acquisition card in Lhasa, a gap of $286 \mathrm{hrs}$ was appeared for the period from 25 November to 7 December 2011. Related information from the SGs at the three stations is given in Table 1. We adopted the same data acquisition systems for these stations to automatically record changes in tidal gravity, barometric pressure, and temperature with a sampling rate of $1 \mathrm{~s}$, respectively.

Similar to a spring gravimeter, the SG is also a relative instrument. Its original feedback output is given in voltage to describe relative gravity changes. Each SG's scale factor (i.e., the transfer function from output voltage to gravity value) must be determined accurately using a strategy of co-site comparison between the SGs and the FG-5 absolute gravimeter, by Micro-g LaCoste Company (Lafayett, CO, USA) (Hinderer et al. 1991; Sun et al. 2001; Amalvict et al. 2002; Riccardi et al. 2012; Van Camp et al. 2016). We conducted this calibration from 18 to 23 September 2013 in Lhasa; from 23 to 25 July 2014 in Lijiang; and from 20 to 24 June 2013 in Wuhan. Figure 2 compares the measurements taken with the SGs and the FG-5 at these three stations.

As seen in Fig. 2, the dispersions in the FG-5 measurements are relatively large and the curves describing the SG measurements are much smoother and have higher precision. Using a linear regression technique, we evaluated the scale factors of the SGs, which were given as follows: $-92.393 \pm$ $0.037 \mu \mathrm{Gal} \mathrm{V}^{-1}$ in Wuhan, $-96.332 \pm 0.061 \mu \mathrm{Gal} \mathrm{V}^{-1}$ in Lijiang, and $-77.129 \pm 0.046 \mu \mathrm{Gal} \mathrm{V}^{-1}$ in Lhasa. Compared with a former scale factor of $-77.736 \pm 0.041 \mu \mathrm{Gal} \mathrm{V}^{-1}$ in Lhasa

Table 1. Basic information of the SG stations and co-site calibration.

\begin{tabular}{ccccccccc}
\hline Stations & Latitude $\left({ }^{\circ} \mathbf{N}\right)$ & Longitude $\left({ }^{\circ} \mathbf{E}\right)$ & Altitude $(\mathbf{m})$ & SG model & Comments & FG5 code & Duration & Valid drops \\
\hline Wuhan & 30.516 & 114.49 & 89.3 & OSG065 & continuous & FG5-112 & $73 \mathrm{~h}$ & 10950 \\
Lijiang & 26.896 & 100.232 & 2435.0 & OSG066 & continuous & FG5-232 & $46.5 \mathrm{~h}$ & 9300 \\
Lhasa & 29.645 & 91.035 & 3632.3 & OSG057 & $286 \mathrm{~h}$ gap & FG5-246 & $120 \mathrm{~h}$ & 18000 \\
\hline
\end{tabular}
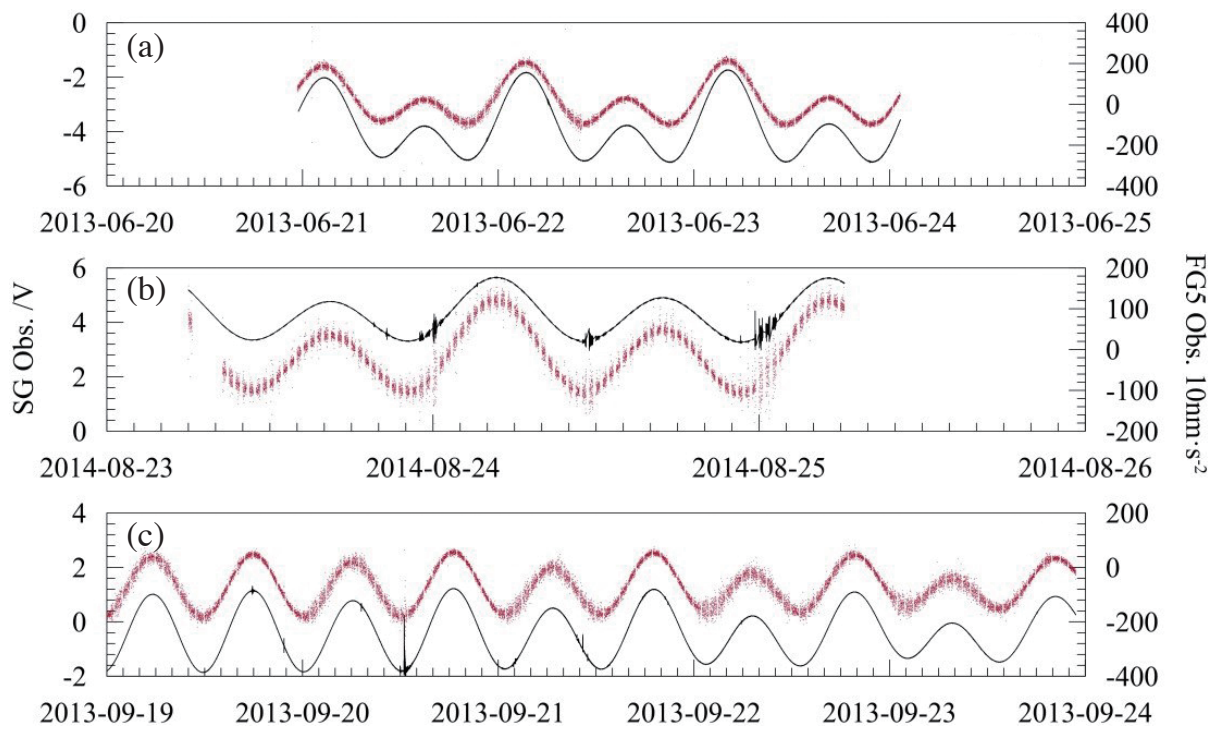

Fig. 2. Comparison of measurements with SGs (black curves) and FG5 (red +) in (a) Wuhan, (b) Lijiang, and (c) Lhasa. 
that was determined in a previous calibration (Chen et al. 2013), our scale factor was about $0.8 \%$ lower. The former scale factor was estimated by co-site comparison between the SG and Lacoste-Romberg (LCR) spring gravimeter (Earth Tidal model, No.20, ET20), by Micro-g LaCoste Company (Lafayett, CO, USA). The scale factor of this LCR-ET20 was obtained at the Wuhan international tidal gravity benchmark station (Xu et al. 2000). In fact, the gravity difference is extremely large because of the significant altitude difference at both Lhasa and Wuhan stations.
Using a remove-restore technique, we treated the original SG records with T-Soft, a graphic and interactive software package (Vauterin 1998). We removed some bad records, such as spikes, steps, and large-amplitude vibrations caused by earthquakes, we modified the offsets; and interpolated small gaps. We used a low-pass filter to transform the $1 \mathrm{~s}$ sampled data series to the hourly ones. The resultant data series of tidal gravity changes are presented in Fig. 3 . We also treated the original station pressure records according to the same procedures (see also Fig. 4).

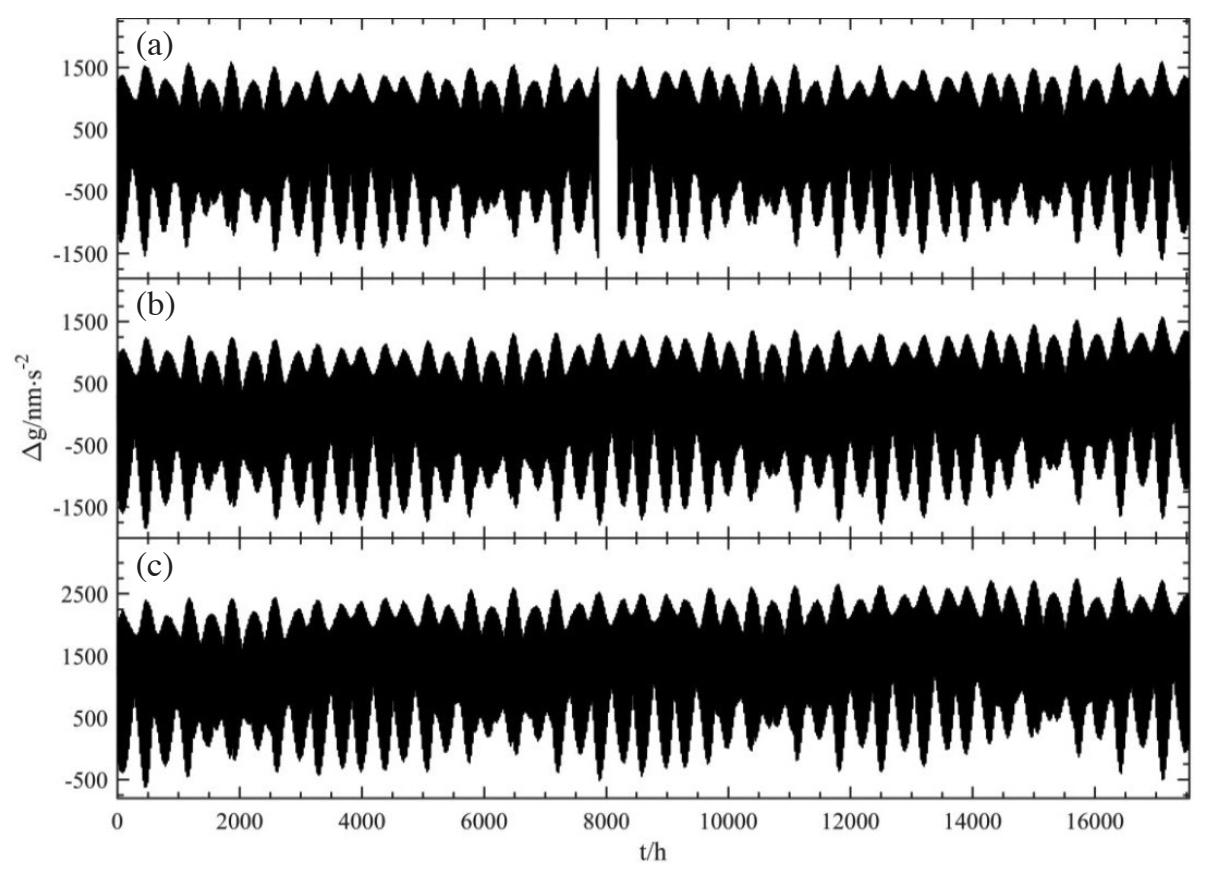

Fig. 3. Temporal gravity variations recorded with the SGs at (a) Lhasa, (b) Lijiang, and (c) Wuhan.

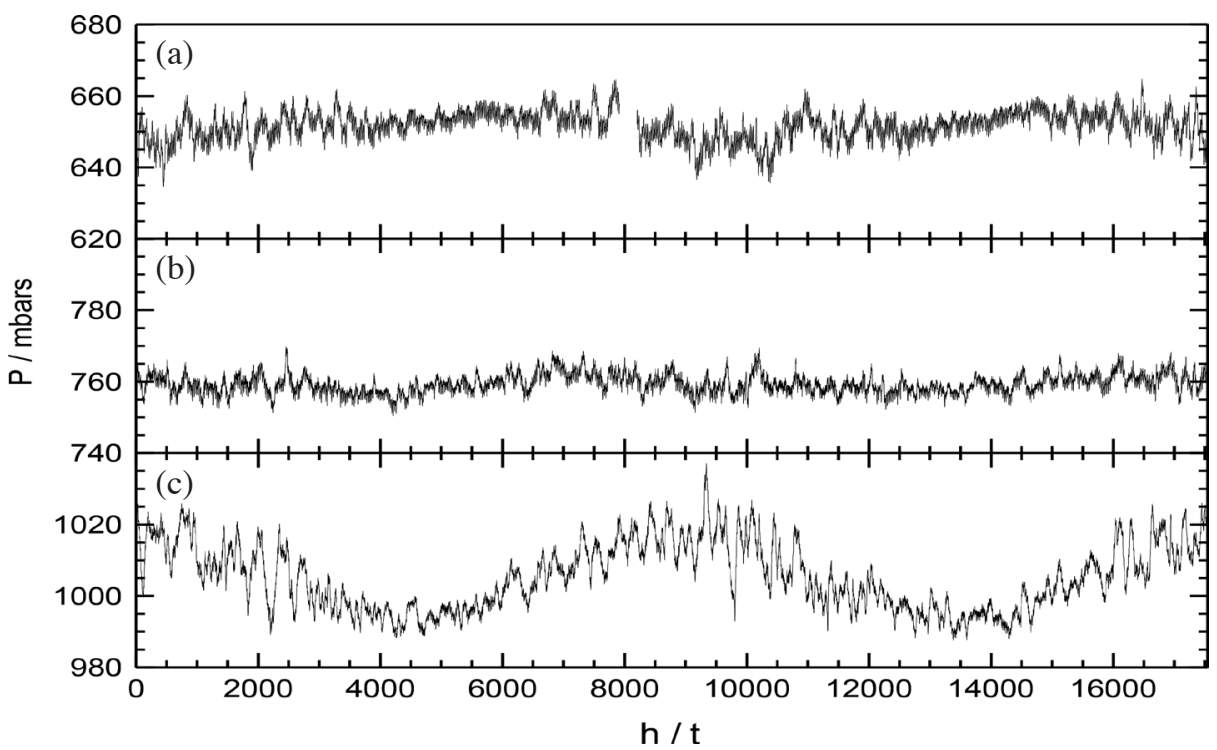

Fig. 4. Temporal pressure variations recorded at stations in (a) Lhasa, (b) Lijiang, and (c) Wuhan. 


\section{TIDAL GRAVITY OBSERVATIONS WITH THE SGS}

After careful data preprocessing, we performed the harmonic analysis using the software package Eterna 3.30 (Wenzel 1996), as recommended by the International Center for Earth Tides. We retrieved accurate tidal gravimetric parameters, including amplitude factors, phase lags, and their corresponding error estimation (see Table 2). For the harmonic analysis, we adopted the high-precision tide-generating potential developed by Hartmann and Wenzel (1995).

Previous studies have shown that the loading effects of atmospheric pressure are the main sources of noise in tidal gravity observations and that more than $90 \%$ of the signals come from an area within $50 \mathrm{~km}$ surrounding the station. Previous studies also showed that the barometric gravity

Table 2. Observations of gravity tides from the SGs at stations in Lhasa, Lijiang, and Wuhan.

\begin{tabular}{|c|c|c|c|c|c|c|c|c|}
\hline \multirow{3}{*}{$\begin{array}{l}\text { Wave } \\
\mathrm{SGQ}_{1}\end{array}$} & \multirow{2}{*}{\multicolumn{2}{|c|}{$\begin{array}{l}\text { Frequency Range } \\
\text { (cpd) }\end{array}$}} & \multicolumn{2}{|c|}{ Lhasa } & \multicolumn{2}{|c|}{ Lijiang } & \multicolumn{2}{|c|}{ Wuhan } \\
\hline & & & \multirow{2}{*}{$\begin{array}{c}\boldsymbol{\delta} \\
1.16352 \pm 0.00735\end{array}$} & \multirow{2}{*}{$\begin{array}{c}\Delta \phi\left(^{\circ}\right) \\
-0.1160 \pm 0.3620\end{array}$} & \multirow{2}{*}{\begin{tabular}{|c|}
$\boldsymbol{\delta}$ \\
$1.17291 \pm 0.02196$
\end{tabular}} & \multirow{2}{*}{$\begin{array}{c}\Delta \phi\left(^{\circ}\right) \\
-1.1243 \pm 1.0748\end{array}$} & \multirow{2}{*}{$\begin{array}{c}\boldsymbol{\delta} \\
1.16238 \pm 0.03710\end{array}$} & \multirow{2}{*}{$\begin{array}{c}\Delta \phi\left(^{\circ}\right) \\
1.3503 \pm 1.8272\end{array}$} \\
\hline & 0.721499 & 0.833113 & & & & & & \\
\hline $2 \mathrm{Q}_{1}$ & 0.851181 & 0.859691 & $1.16297 \pm 0.00233$ & $1.0821 \pm 0.1150$ & $1.17727 \pm 0.00715$ & $0.4860 \pm 0.3480$ & $1.19690 \pm 0.01206$ & $0.5953 \pm 0.5787$ \\
\hline$\sigma_{1}$ & 0.860895 & 0.870024 & $16433 \pm 0.00199$ & $0.5547 \pm 0.0982$ & $1.18834 \pm 0.00601$ & $-0.3479 \pm 0.2899$ & $1.17352 \pm 0.01017$ & $0.1605 \pm 0.4965$ \\
\hline $\mathrm{Q}_{1}$ & 0.887326 & 0.896130 & $1.16543 \pm 0.00032$ & $0.2361 \pm 0.0158$ & $1.17210 \pm 0.00099$ & $-0.1968 \pm 0.0486$ & $1.18001 \pm 0.00168$ & $-0.2461 \pm 0.0817$ \\
\hline$\rho_{1}$ & 0.897806 & 0.906316 & $16229 \pm 0.00178$ & $.2609 \pm 0.0872$ & $1.17750 \pm 0.00566$ & $-0.3377 \pm 0.2751$ & $1.18681 \pm 0.00958$ & $-0.4056 \pm 0.46$ \\
\hline $\mathrm{O}_{1}$ & 0.921940 & 0.930450 & $1.16101 \pm 0.00006$ & $0.0107 \pm 0.0032$ & $1.16564 \pm 0.00020$ & $-0.3911 \pm 0.0099$ & $1.17571 \pm 0.00034$ & $-0.4804 \pm 0.0166$ \\
\hline$\tau_{1}$ & 0.931963 & .940488 & $14875 \pm 0.00489$ & $.6679 \pm 0.2435$ & $.16519 \pm 0.00975$ & $0.1925 \pm 0.4793$ & $.19875 \pm 0.01654$ & $-0.5764 \pm 0.7907$ \\
\hline $\mathrm{NO}_{1}$ & 0.958085 & 0.966757 & $15385 \pm 0.00076$ & $-0.1691 \pm 0.0376$ & $1.16429 \pm 0.00198$ & $-0.3240 \pm 0.0974$ & $1.17082 \pm 0.00337$ & -0.4827 \\
\hline$\chi_{1}$ & 0.968564 & 0.974189 & $.16157 \pm 0.00439$ & $-0.0847 \pm 0.2168$ & $1.16427 \pm 0.01380$ & $-0.0422 \pm 0.6792$ & $1.15742 \pm 0.02335$ & $-0.8393 \pm$ \\
\hline$\pi_{1}$ & 0.989048 & 0.995144 & $.14733 \pm 0.00330$ & $-0.7982 \pm 0.1630$ & $1.15160 \pm 0.00613$ & $-0.7642 \pm 0.3048$ & $1.16863 \pm 0.01039$ & $-2.2225 \pm 0.5096$ \\
\hline $\mathrm{P}_{1}$ & 0.996967 & 0.998029 & $14934 \pm 0.00024$ & $.0030 \pm 0.0122$ & $1.15228 \pm 0.00036$ & $-0.3484 \pm 0.0179$ & $1.16237 \pm 0.00061$ & $-0.5722 \pm 0.0$ \\
\hline $\mathrm{S}_{1}$ & 0.999852 & 1.000148 & $59167 \pm 0.02187$ & $19.0573 \pm 0.7547$ & $1.57459 \pm 0.02697$ & $20.8111 \pm 1.2692$ & $1.28292 \pm 0.04101$ & $16.2532 \pm 1.8936$ \\
\hline $\mathrm{K}_{1}$ & 1.001824 & 1.003652 & $13550 \pm 0.00009$ & $0.0597 \pm 0.0046$ & $.13924 \pm 0.00014$ & $-0.3328 \pm 0.0068$ & $1.14887 \pm 0.00023$ & $-0.5787 \pm 0.0$ \\
\hline$\psi_{1}$ & 1.005328 & 1.005624 & $24723 \pm 0.01037$ & $0.1047 \pm 0.4762$ & $1.24746 \pm 0.01543$ & $-0.6100 \pm 0.7089$ & $1.21339 \pm 0.02604$ & $-3.1292 \pm 1.2285$ \\
\hline$\phi_{1}$ & 94 & 90 & $17340+0004$ & $06506+02134$ & $117547+0008$ & 3970 & 1.19630 & 0.87 \\
\hline$\theta_{1}$ & 1.028549 & 1.034468 & $15345 \pm 0.00449$ & $.1308 \pm 0.2230$ & $1.15104=$ & 0.07 & $1.17359 \pm 0.02302$ & -0.964 \\
\hline $\mathrm{J}_{1}$ & 1.036291 & 1.044801 & $.15700 \pm 0.00083$ & $0.0221 \pm 0.0414$ & $1.16081 \pm 0.00254$ & $-0.2215 \pm 0.1252$ & $1.16309 \pm 0.00427$ & $-0.6120 \pm 0.2105$ \\
\hline $\mathrm{SO}_{1}$ & 1.064840 & 1.071084 & $17138 \pm 0.00515$ & $-0.2424 \pm 0.2513$ & $1.13268 \pm 0.01588$ & $-1.9805 \pm 0.8026$ & $1.07640 \pm 0.02681$ & $0.2419 \pm 1.4269$ \\
\hline $\mathrm{OO}_{1}$ & 82 & 1.0 & 1561 & $00876+0$ & 19 & -0.2 & 1048 & -1.99 \\
\hline$v_{1}$ & 1.099160 & 1.216398 & $14337 \pm 0.00754$ & $.4660 \pm 0.3773$ & $1.17943 \pm 0.03064$ & $1.2424 \pm 1.4889$ & $1.10020 \pm 0.05181$ & $1.6849 \pm 2.6973$ \\
\hline$\varepsilon_{2}$ & 30 & 0 & $17720 \pm 0.002$ & $.1688 \pm 0.1288$ & $16491 \pm 0.0$ & $0.0552 \pm 0$ & $1.17971 \pm 0$ & $0.3652 \pm 0.3$ \\
\hline $2 \mathrm{~N}_{2}$ & 1.853919 & 1.862429 & $1.17280 \pm 0.00085$ & $-0.6763 \pm 0.0415$ & $1.16892 \pm 0.00147$ & $-0.5198 \pm 0.0723$ & $1.18109 \pm 0.00223$ & -0.2860 \\
\hline$\mu_{2}$ & 3636 & 3 & $16785 \pm 0.00068$ & $.4769 \pm 0.0335$ & $16886 \pm 0.0$ & $-0.4235 \pm 0.0610$ & $1.17440 \pm 0.00189$ & $-0.0733 \pm 0.0$ \\
\hline $\mathrm{N}_{2}$ & 1.888386 & 1.896749 & $1.16463 \pm 0.00011$ & $-0.5937 \pm 0.0053$ & $1.16649 \pm 0.00020$ & $-0.4095 \pm 0.0098$ & $1.17481 \pm 0.00030$ & $-0.4797 \pm 0.0147$ \\
\hline$v_{2}$ & 1.897953 & 1.906463 & $16313 \pm 0.00055$ & $-0.6240 \pm 0.0270$ & $1.16487 \pm 0.00105$ & $-0.4109 \pm 0.0519$ & $1.17606 \pm 0.00160$ & $-0.5875 \pm 0.0780$ \\
\hline $\mathrm{M}_{2}$ & 1.923765 & 1.942754 & $1.16289 \pm 0.00002$ & $-0.4388 \pm 0.0010$ & $1.16575 \pm 0.00004$ & $-0.3505 \pm 0.0019$ & $1.17159 \pm 0.00006$ & $-0.4376 \pm 0.0028$ \\
\hline$\lambda_{2}$ & 58232 & 963709 & $1.16085 \pm 0.00276$ & $-0.5854 \pm 0.1364$ & $1.15152 \pm 0.00513$ & $-0.4522 \pm 0.2554$ & $1.16563 \pm 0.0078$ & $-0.2128 \pm 0.3835$ \\
\hline $\mathrm{L}_{2}$ & 1.965826 & 1.976927 & $1.16198 \pm 0.00054$ & $-0.3162 \pm 0.0264$ & $1.15936 \pm 0.00159$ & $-0.3678 \pm 0.0788$ & $1.15828 \pm 0.00242$ & $-0.5863 \pm 0.1196$ \\
\hline $\mathrm{T}_{2}$ & 1.991786 & 88 & $1.104 J 1 \pm 0.00000$ & $-0.8526 \pm 0.0433$ & $1.16775 \pm 0.00144$ & $-0.8892 \pm 0.0729$ & $1.17108 \pm 0.00218$ & $-1.0462 \pm 0.1092$ \\
\hline $\mathrm{S}_{2}$ & 1.999705 & 2.000767 & $1.15696 \pm 0.00006$ & $-0.6657 \pm 0.0036$ & $1.15925 \pm 0.00010$ & $-0.6126 \pm 0.0079$ & $1.16550 \pm 0.00015$ & $-0.6790 \pm 0.0100$ \\
\hline $\mathrm{K}_{2}$ & 2.00259 & .013690 & $1.15772 \pm 0.00017$ & $-0.3910 \pm 0.0085$ & $1.16141 \pm 0.00041$ & $-0.3456 \pm 0.0203$ & $1.16565 \pm 0.00062$ & $-0.4493 \pm 0.0309$ \\
\hline$\eta_{2}$ & 2.031287 & 2.047391 & $1.15981 \pm 0.00316$ & $-0.3040 \pm 0.1560$ & $1.15431 \pm 0.00833$ & $-0.2136 \pm 0.4136$ & $1.14639 \pm 0.01262$ & $-0.3930 \pm 0.6309$ \\
\hline $2 \mathrm{~K}_{2}$ & 2.067578 & 2.182844 & $1.15946 \pm 0.00943$ & $0.0717 \pm 0.4658$ & $1.14342 \pm 0.03094$ & $-1.5935 \pm 1.5503$ & $1.20064 \pm 0.04705$ & $1.3665 \pm 2.2452$ \\
\hline $\mathrm{MN}_{2}$ & 2.753243 & 2.869714 & $1.07127 \pm 0.00283$ & $-0.0565 \pm 0.1515$ & $1.07699 \pm 0.00512$ & $-0.4391 \pm 0.2722$ & $1.08348 \pm 0.0076$ & $-0.5734 \pm 0.4021$ \\
\hline $\mathrm{M}_{3}$ & 2.892639 & 3.081255 & $1.07333 \pm 0.00077$ & $-0.0299 \pm 0.0411$ & $1.07233 \pm 0.00146$ & $-0.0232 \pm 0.0782$ & $1.07993 \pm 0.00218$ & $-0.1496 \pm 0.1156$ \\
\hline
\end{tabular}


admittance (i.e., the transfer function from the local pressure to its resultant gravity change) is in a range from -0.3 to $-0.4 \mu \mathrm{Gal} \mathrm{hPa}^{-1}$ and about $-0.3317 \mu \mathrm{Gal} \mathrm{hPa}^{-1}$ on average (Merriam 1992; Sun 1997; Kroner and Jentzsch 1999; Boy et al. 2002, 2006; Meurers 2012). As show in Fig. 4, the main components of pressure variations were concentrated in the $\mathrm{D}$ and SD bands. However, an annual signal can be found for station Wuhan, but no such variation was found for the stations in Lhasa and Lijiang, this is the special phenomena in plateau region. The peak-to-peak magnitude was as large as $49.48 \mathrm{hPa}$ in Wuhan, and the dominant signals concentrated at the annual band. The pressure changes behaved primarily in short periods in D and SD bands in Lhasa and Lijiang, however, and the peak-to-peak magnitudes were 30.18 and $19.31 \mathrm{hPa}$, respectively. Using a linear regression technique between tidal gravity residuals and station pressure, we determined the atmospheric gravity admittances, they were as follows: $-0.3721 \pm 0.0006 \mu \mathrm{Gal} \mathrm{hPa}^{-1}$ in Lhasa, -0.3603 $\pm 0.0022 \mu \mathrm{Gal} \mathrm{hPa}^{-1}$ in Lijiang, and $-0.3376 \pm 0.0027 \mu \mathrm{Gal}$ $\mathrm{hPa}^{-1}$ in Wuhan. These results were in the range of theoretical simulations, and they increased with station altitude. After removing local pressure effects, the standard deviations in tidal gravity band decreased significantly by 77,37 , and $27 \%$ for the stations in Lhasa, Lijiang, and Wuhan, respectively.

When comparing the numerical results of tidal gravity amplitudes for different stations, we found that the observed amplitudes were slightly different. For example, the amplitude of wave $\mathrm{O}_{1}$, which is the most stable wave with the highest precision in the $\mathrm{D}$ band, was $31.93 \mu \mathrm{Gal}$ in Wuhan, which was about $2.9 \%$ larger than that obtained in Lhasa and was about $8.5 \%$ larger than that obtained in Lijiang. The precision of amplitude factor is $0.006 \%$ in Lhasa and $0.03 \%$ in Wuhan. In contrast, the amplitude of wave $\mathrm{M}_{2}$, which is the most stable wave with the highest precision in the SD band, was $65.30 \mu \mathrm{Gal}$ in Wuhan, which is about $1.1 \%$ lower than that obtained in Lhasa and about $6.2 \%$ less than that obtained in Lijiang. The precision of amplitude factor is $0.002 \%$ in Lhasa and $0.006 \%$ in Wuhan. These numerical results indicated that the precision of tidal gravity observations measured with the SGs was very high. The standard deviation in the tidal band was $0.0489 \mu \mathrm{Gal}$ in Lhasa, $0.1260 \mu \mathrm{Gal}$ in Lijiang, and $0.1694 \mu \mathrm{Gal}$ in Wuhan. For the main tidal waves with amplitudes exceeding $10 \mu \mathrm{Gal}$, the precision of the amplitude factors was better than $0.05 \%$, and for all other tidal waves with amplitudes exceeding $5 \mu \mathrm{Gal}$, the precision is better than $0.14 \%$. Therefore, these findings indicate the tidal gravity observations in this study can distinguish miniscule characteristics in the Earth's response to the tide-generating forces.

Table 2 shows that the amplitude factor $\delta$ of $S_{1}$ had relatively large differences for the different stations: 1.59167 in Lhasa, 1.57459 in Lijiang, and 1.28292 in Wuhan. In addition, their phase differences $\Delta \phi$ were extremely abnormal. This analysis showed that these abnormalities were the result of the solar heating, which contributed to changes in local atmospheric pressure and expansion or shrinking of local rock. Such perturbation relating to solar heating was mixed in the SG observations for wave $S_{1}$. The solar heating effects were much more conspicuous in Lhasa and Lijiang compared with the effects in Wuhan. Meanwhile, the solar heating effects were much weaker for the station in Wuhan.

The tidal gravity results are given here for the first time for station Lijiang. We also found that the tidal gravity parameters determined in Lhasa and Wuhan were in good agreement with those obtained in previous studies (Sun et al. 2005b, 2013; Wei et al. 2012; Xu et al. 2012, 2014). Compared with the previous findings in Lhasa, the amplitude factors $\delta$ of the main tidal waves were about $0.8 \%$ larger as a result of evaluation bias in the scale factors in the previous studies, whereas the phase differences $\Delta \phi$ were nearly consistent.

\section{LOADING CORRECTION OF OCEANIC TIDES}

The global loading of oceanic tides will lead to obvious tidal gravity changes with same spectral pattern. To obtain a pure response of such tide-generating forces, we had to completely remove them. On the basis of the classical loading theory (Farrell 1972), we simulated the tidal gravity loading vectors, including magnitude $L$ and phase $\lambda$, using a global convolution technique that loaded Green's functions and oceanic tide models (Agnew 1997; Boy et al. 2003; Sun et al. 2005a).

Schwiderski developed the first available global model of oceanic tides in 1980 based on numerical solution of the tidal movement equations, while considering global tidal gauge data (Schwiderski 1980). In recent decades, with the development of satellite altimetry and additional tidal gauge data accumulation, many new oceanic tidal models, have been successfully developed. Previous studies have shown that the NAO99 global oceanic model (National Astronomical Observatory, Japan) will provide with the best results for stations in the Asian region (Sun et al. 2005a; Xu et al. 2014). This model combines five-year data of Topex/Poseidon altimetry and density tidal gauge data recorded along the west Pacific area, in particular, areas near the Japanese islands (Matsumoto et al. 2000). The model contains cotidal charts of seven $\mathrm{D}$ waves (i.e., $\mathrm{Q}_{1}, \mathrm{O}_{1}, \mathrm{M}_{1}, \mathrm{P}_{1}, \mathrm{~K}_{1}, \mathrm{~J}_{1}$, and $\mathrm{OO}_{1}$ ) and nine $\mathrm{SD}$ waves (i.e., $2 \mathrm{~N}_{2}, \mu_{2}, \mathrm{~N}_{2}, \mathrm{v}_{2}, \mathrm{M}_{2}, \mathrm{~L}_{2}, \mathrm{~T}_{2}$, $\mathrm{S}_{2}$, and $\mathrm{K}_{2}$ ) with a spatial resolution of $0.5^{\circ} \times 0.5^{\circ}$. By using these co-tidal charts, we then evaluated the loading vector by considering the regional co-tides near China for waves $\mathrm{O}_{1}, \mathrm{~K}_{1}, \mathrm{M}_{2}$, and $\mathrm{S}_{2}$ (Fang et al. 1999). The numerical results are presented in Table 3.

We found that the loading effects were much lower for stations located in the Tibetan Plateau when compared with the effects at the station Wuhan. As seen in Table 3, the loading effects were at a level less than $1 \mu \mathrm{Gal}$ at all three 
SG stations. Considering the tidal waves of the largest amplitudes as examples, the loading value was of $0.613 \mu \mathrm{Gal}$ in wave $\mathrm{O}_{1}$, which was about $1.9 \%$ of its observed tidal gravity amplitude in Wuhan. This loading value was also about 4.9 and 2.3 times larger than the ones in Lhasa and Lijiang. The loading effects contributed to a disturbance of 0.580 $\mu \mathrm{Gal}$ in wave $\mathrm{K}_{1}$, which was about $1.5 \%$ of its observed tidal gravity amplitude in Wuhan, and the loading value was about 3.3 and 4.6 times larger than the disturbances in Lhasa and Lijiang. The loading effects resulted in a disturbance of $0.863 \mu \mathrm{Gal}$ in wave $\mathrm{M}_{2}$, which was about $1.3 \%$ of its observed tidal gravity amplitude in Wuhan, and this loading value was about 2.1 and 2.4 times larger than the ones in Lhasa and Lijiang. After subtracting the loading effects from these observations, we then obtained the gravimetric parameters describing the Earth's pure response to the tidegenerating forces (see also Table 3).

From Table 3, in Wuhan, it is evident that after removal of the oceanic loading signals, the gravimetric amplitude factors significantly decreased by $1.9,1.2$, and $1.2 \%$ for main tidal waves $\mathrm{O}_{1}, \mathrm{~K}_{1}$, and $\mathrm{M}_{2}$, respectively, and decreased by $0.2-3.8 \%$ for the other tidal waves. In Lhasa, the amplitude factors $\delta$ for the main waves $\mathrm{O}_{1}, \mathrm{~K}_{1}$, and $\mathrm{M}_{2}$ decreased by less than $0.3 \%$, but changed only slightly for the other tidal waves. In Lijiang, the amplitude factor for wave $\mathrm{O}_{1}$ decreased by $0.8 \%$, and by less than $0.2 \%$ for the other tidal waves.

Our results show that the resultant phase differences $\Delta \phi$ changed significantly and were more reasonable after oceanic loading correction. Therefore, we concluded that oceanic loading effects should be considered when observing the high precision of tidal gravity and explaining the geophysical and geodynamical phenomena.

After removing the oceanic loading signals, the tidal gravity amplitude factors in Wuhan were in good agreement with the results from the theoretical simulation (Wahr 1981; Dehant et al. 1999; Mathews 2001) and also were in good agreement with the results from the experimental model (Sun et al. 2003; Xu et al. 2004). After considering the Earth's rotation, ellipticity, and inelasticity, Mathews (2001) theoretically simulated the Earth's response to the tide-generating forces in 2001. In his simulation he modified some parameters in the preliminary reference Earth model to match the nutation measurements (Mathews 2001).

Comparing the tidal gravity amplitude factors with theoretical simulation for the station in Wuhan, we found that the discrepancy was less than $0.04 \%$ on average for the main D waves, in which the discrepancy was $0.05 \%$ for wave $\mathrm{O}_{1}$ and $0.02 \%$ for wave $\mathrm{K}_{1}$, which was in the range of the SG's measuring precision. Conversely, the average discrepancy was less than $0.14 \%$ for the main SD waves, in which the discrepancy was $0.15 \%$ for wave $M_{2}$ and $0.24 \%$ for wave $\mathrm{S}_{2}$.

The diagram comparing the amplitude factors for sev- eral main waves in the D and SD bands after oceanic loading correction is shown in Fig. 5. We found that the amplitude factors did not have remarkable difference for the stations in the Tibetan Plateau as in Lhasa and Lijiang, and it was only at the level of $0.1 \%$ or less for most tidal waves. These factors, however, were significantly larger than those obtained in Wuhan. The analysis showed that in the D band, the amplitude factors were $0.25 \%$ (Lhasa) and $0.30 \%$ (Lijiang) larger than those measured in Wuhan, on average. For example, for wave $\mathrm{O}_{1}$, the amplitude factor was about $0.31 \%$ (Lhasa) and $0.23 \%$ (Lijiang) larger than that in Wuhan, and for wave $\mathrm{K}_{1}$, it was about $0.32 \%$ (Lhasa) and $0.37 \%$ (Lijiang) larger than that in Wuhan. In the SD band, the amplitude factors were $0.61 \%$ (Lhasa) and $0.48 \%$ (Lijiang) larger than those measured in Wuhan, on average. As an example, for wave $\mathrm{M}_{2}$, the amplitude factor was about $0.41 \%$ (Lhasa) and $0.43 \%$ (Lijiang) larger than that in Wuhan, and for wave $\mathrm{S}_{2}$, it was about $0.21 \%$ (Lhasa) and $0.16 \%$ (Lijiang) larger than that in Wuhan.

As we know, the main tidal waves $\mathrm{O}_{1}, \mathrm{~K}_{1}, \mathrm{M}_{2}$, and $\mathrm{S}_{2}$ have the highest signal-to-noise ratios, and the observations for wave $\mathrm{S}_{2}$ may also have been contaminated more or less by solar heating radiation. The mean gravimetric amplitude factors of $\mathrm{O}_{1}, \mathrm{~K}_{1}$, and $\mathrm{M}_{2}$, retrieved in Lhasa and Lijiang were about $0.27,0.34$, and $0.42 \%$ larger, respectively, than those measured in Wuhan. The mean difference was about $0.34 \%$, which we regarded as a local influence of the Tibetan Plateau. Of course, these finding may reflect the comprehensive effects resulting from an elastic property of an extremely thick crust, the complicated structure in the regional lithosphere, and the strong heterogeneity and anisotropy of the medium.

\section{DISCUSSION AND CONCLUSIONS}

To study the regional response of the Tibetan Plateau to tide-generating forces, we used two-year continuous tidal gravity measurements recorded by SGs at three stations in China, Lhasa located in the central-southern area of the Tibetan Plateau; Lijiang, located in the eastern-southern edge of the Tibetan Plateau; and Wuhan, located in the plain, a central Yangzi platform and far away from the plateau. We accurately scaled all of the SGs by comparing the measurements with FG5 at a scaling precision as of better than $0.06 \%$. The determined precision of tidal gravity amplitude factors was better than $0.05 \%$ for the main tidal waves and was better than $0.14 \%$ for the other tidal waves.

We considered the effects of atmospheric pressure on tidal gravity observations. The results indicated that these effects are significantly different between the station in the Tibetan Plateau (Lhasa and Lijiang) and the station in the plain (Wuhan). The barometric pressure change behaved mainly as short-period vibrations in D and SD bands in all three stations, but it also behaved mainly as annual variations 
of a large magnitude in Wuhan. We found that the barometric gravity admittance showed an increasing tendency with station altitude. After we removed pressure influence, the standard deviation of tidal gravity decreased significantly.

Considering the global oceanic models and regional models near China, the oceanic gravity loading signals was in a range from $0.2-1.9 \%$ of the amplitude of the tidal gravity waves in Wuhan, but they were much lower in Lhasa and Lijiang. After removing these loading effects, the gravimetric amplitude factors measured in Lhasa decreased by about $0.3 \%$ on average, and decrease significantly by about $1.2 \%$ on average in Wuhan.
We found that no significant differences existed in the amplitude factors measured in Lhasa and Lijiang, but a relative remarkably difference was found when compared with the factors measured in Wuhan. The mean gravimetric amplitude factors of waves $\mathrm{O}_{1}, \mathrm{~K}_{1}$, and $\mathrm{M}_{2}$ were about $0.27,0.34$, and $0.42 \%$ larger than those measured in Wuhan, respectively. The tidal gravity anomaly was about $0.34 \%$, which we regarded as a local phenomenon unique to the Tibetan Plateau.

Previous studies have shown that following the collision between the India Plate and Eurasia Plate, deep substance began to adjust and move. This resulted not only in

Table 3. Oceanic loading vectors and gravimetric parameters after removal of oceanic loading effects.

\begin{tabular}{|c|c|c|c|c|c|c|c|c|c|c|c|c|c|}
\hline \multirow{2}{*}{ Wave } & \multicolumn{4}{|c|}{ Lhasa } & \multicolumn{4}{|c|}{ Lijiang } & \multicolumn{5}{|c|}{ Wuhan } \\
\hline & $L(\mu \mathrm{Gal})$ & $\lambda\left({ }^{\circ}\right)$ & $\delta$ & $\Delta \phi\left(^{\circ}\right)$ & $L(\mu \mathrm{Gal})$ & $\lambda\left({ }^{\circ}\right)$ & $\delta$ & $\Delta \phi\left(^{\circ}\right)$ & $L(\mu \mathrm{Gal})$ & $\lambda\left({ }^{\circ}\right)$ & $\delta$ & $\Delta \phi\left(^{\circ}\right)$ & $\delta^{*}$ \\
\hline $\mathrm{Q}_{1}$ & 0.04920 & 44.18 & 1.15852 & -0.09 & 0.06320 & -7.30 & 1.15903 & -0.12 & 0.13540 & -5.32 & 1.15408 & -0.13 & 1.15410 \\
\hline $\mathrm{O}_{1}$ & 0.12454 & 33.23 & 1.15711 & -0.12 & 0.26481 & -25.62 & 1.15609 & -0.17 & 0.61361 & -10.64 & 1.15348 & -0.28 & 1.15401 \\
\hline $\mathrm{M}_{1}$ & 0.00570 & -78.42 & 1.15330 & -0.04 & 0.02020 & -68.91 & 1.16058 & 0.15 & 0.04480 & -32.87 & 1.15316 & 0.08 & 1.15351 \\
\hline $\mathrm{P}_{1}$ & 0.03080 & 151.75 & 1.15152 & -0.06 & 0.06730 & -84.35 & 1.15169 & -0.06 & 0.21340 & -35.23 & 1.14852 & -0.09 & 1.14892 \\
\hline $\mathrm{K}_{1}$ & 0.17355 & 135.62 & 1.13880 & -0.10 & 0.12605 & -92.76 & 1.13940 & -0.15 & 0.58050 & -26.46 & 1.13521 & -0.24 & 1.13494 \\
\hline $\mathrm{J}_{1}$ & 0.01810 & 120.09 & 1.16134 & -0.35 & 0.00230 & 174.87 & 1.16197 & -0.23 & 0.02620 & -30.51 & 1.15247 & -0.31 & 1.15619 \\
\hline $\mathrm{OO}_{1}$ & 0.01830 & 106.16 & 1.16062 & -0.67 & 0.00750 & 93.31 & 1.15651 & -0.58 & 0.01150 & -6.67 & 1.16434 & -1.95 & 1.15555 \\
\hline $2 \mathrm{~N}_{2}$ & 0.02120 & -88.38 & 1.17230 & 0.04 & 0.00990 & -74.43 & 1.16712 & -0.21 & 0.02650 & -8.64 & 1.16253 & -0.15 & \\
\hline$\mu_{2}$ & 0.01810 & -92.61 & 1.16828 & 0.03 & 0.01290 & -68.55 & 1.16625 & -0.10 & 0.03260 & -11.67 & 1.15567 & 0.12 & \\
\hline $\mathrm{N}_{2}$ & 0.10940 & -83.86 & 1.16349 & -0.10 & 0.08510 & -63.27 & 1.16312 & -0.08 & 0.18450 & -22.00 & 1.15874 & -0.17 & \\
\hline$v_{2}$ & 0.01880 & -83.93 & 1.16210 & -0.18 & 0.01540 & -59.53 & 1.16125 & -0.11 & 0.03550 & -21.47 & 1.15971 & -0.28 & \\
\hline $\mathrm{M}_{2}$ & 0.41411 & -88.15 & 1.16262 & -0.08 & 0.36386 & -61.54 & 1.16283 & -0.09 & 0.86334 & -27.93 & 1.15787 & -0.08 & 1.15610 \\
\hline $\mathrm{L}_{2}$ & 0.00700 & -104.12 & 1.16303 & -0.11 & 0.00680 & -61.28 & 1.15741 & -0.19 & 0.02120 & -34.26 & 1.14711 & -0.21 & \\
\hline $\mathrm{T}_{2}$ & 0.00640 & -142.89 & 1.16784 & -0.73 & 0.00320 & -98.27 & 1.16800 & -0.79 & 0.01660 & -39.67 & 1.16254 & -0.71 & \\
\hline $\mathrm{S}_{2}$ & 0.14210 & -144.49 & 1.16131 & -0.51 & 0.07549 & -123.45 & 1.16073 & -0.50 & 0.24288 & -45.35 & 1.15886 & -0.35 & \\
\hline $\mathrm{K}_{2}$ & 0.03390 & -147.76 & 1.16170 & -0.27 & 0.01170 & -124.48 & 1.16228 & -0.28 & 0.07420 & -29.82 & 1.15648 & -0.19 & \\
\hline
\end{tabular}

Note: $L$ and $\lambda$ are the oceanic loading magnitude and phase, $\delta$ and $\Delta \phi$ are the amplitude factor and phase differences after loading effects are removed, $\delta *$ stands for the gravimetric amplitude factor simulated theoretically by Mathews (2001), in which the modified preliminary reference Earth model (M-PREM) is adopted and Earth's rotation, ellipticity, and inelasticity are taken into account.

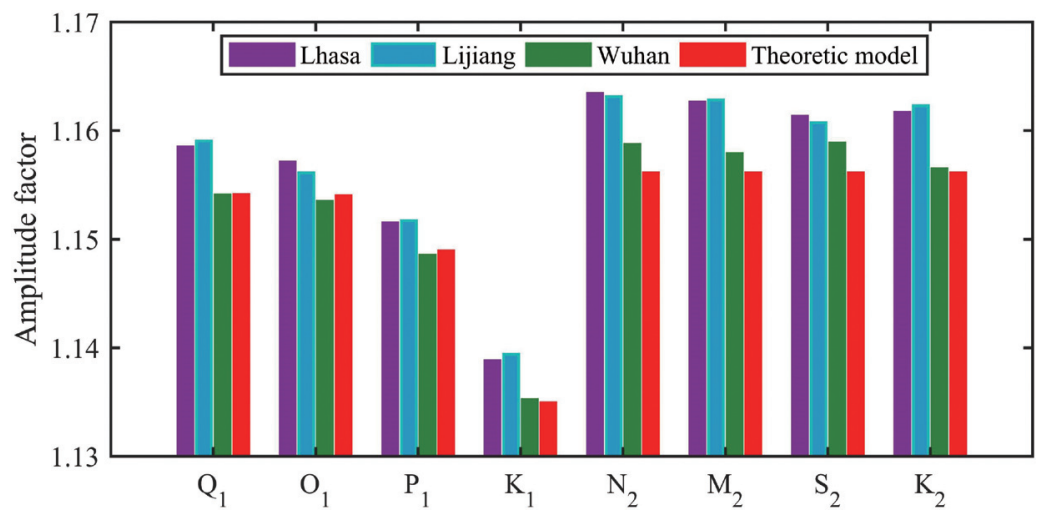

Fig. 5. Comparison of the amplitude factors with Mathews's theoretical model at stations Lhasa, Lijiang, and Wuhan. 
a shortening and thickening of the Tibetan Plateau, but also in the lateral flow of the deep substance in the crust and upper mantle (Teng et al. 2012). On the basis of the stratified velocity structure, especially the special layers beneath the Tibetan Plateau and its adjacent areas, research results have suggested that the crust beneath the Tibetan Plateau is very thick. Therefore, a special deep process and geodynamical environment exists beneath the hinterland of the Tibetan Plateau and periphery.

Because of the SG's advantages of high precision and sensitivity, the tidal gravity observations surely have the potential to distinguish miniscule characteristics in the Earth's regional tidal deformations. Because estimated accuracy of the tidal gravimetric amplitude factors is better than $0.05 \%$, the results given in this study appear to have the potential to reveal the influence of an unusual regional tectonic structure and lithosphere effective elastic thickness.

The tidal gravity observations in Wuhan, however, illustrated the tidal deformation of a "mean Earth". From our numerical results and analysis, we found that the loading effects from oceanic and atmospheric tides should not be used to explain differences in the gravimetric amplitude factors obtained from the stations located in the Tibetan Plateau and in the plain. Jiang et al. (2014) demonstrated that the effective elastic thickness in the central part of Tibetan Plateau was $35-45 \mathrm{~km}$, which was much larger than that in other areas. Therefore, the altitudes of the SGs at the three stations and the thickness and elastic properties of the crust and upper mantle must be responsible for these observed anomalies. In other words, referring to the results given by Shukowsky and Mantovani (1999), the lithosphere effective elastic thickness in the Tibetan Plateau area does have a significant influence on high-precision tidal gravity results.

To draw specific conclusions, however, further investigations are needed, including theoretical simulations of the Earth's deformation under the attraction of the celestial bodies, in which the thickness and complicated structure under the Tibetan Plateau should be considered. More accurate estimations of these gravimetric parameters with longer accumulated SG observations also are necessary.

Acknowledgements The authors would like to express their sincere thanks to Professor XingHua Hao and his group for their careful maintenance of the SG instruments. This work is jointly supported by National Natural Science Foundation of China (Grant Nos. 41621091, 41474062, 41274085, 41574072, and 416740083) and National Key Basic Research Program of China (973 Program, grant No. 2014CB845902).

\section{REFERENCES}

Agnew, D. C., 1997: NLOADF: A program for computing ocean-tide loading. J. Geophys. Res., 102, 5109-5110, doi: 10.1029/96jb03458. [Link]

Amalvict, M., J. Hinderer, P. Gegout, S. Rosat, and D. Crossley, 2002: On the use of AG data to calibrate SG instruments in the GGP network: Example of Strasbourg-J9. Bull. Inform. Marees Terr., 135, 10621-10626.

Boy, J. P., P. Gegout, and J. Hinderer, 2002: Reduction of surface gravity data from global atmospheric pressure loading. Geophys. J. Int., 149, 534-545, doi: 10.1046/j.1365-246x.2002.01667.x. [Link]

Boy, J. P., M. Llubes, J. Hinderer, and N. Florsch, 2003: A comparison of tidal ocean loading models using superconducting gravimeter data. J. Geophys. Res., 108, doi: 10.1029/2002JB002050. [Link]

Boy, J. P., R. Ray, and J. Hinderer, 2006: Diurnal atmospheric tide and induced gravity variations. J. Geodyn., 41, 253-258, doi: 10.1016/j.jog.2005.10.010. [Link]

Chen, X., H. Sun, H. Xu, J. Xu, X. Hao, and M. Liu, 2013: Determination of the calibration factor of superconducting gravimeter 057 at the Lhasa station: A frequencydomain approach. Terr. Atmos. Ocean. Sci., 24, 629636, doi: 10.3319/TAO.2012.12.03.02(TibXS). [Link]

Crossley, D. and J. Hinderer, 2009: A review of the GGP network and scientific challenges. J. Geodyn., 48, 299304, doi: 10.1016/j.jog.2009.09.019. [Link]

Dehant, V., P. Defraigne, and J. M. Wahr, 1999: Tides for a convective Earth. J. Geophys. Res., 104, 1035-1058, doi: 10.1029/1998jb900051. [Link]

Doodson, A. T., 1921: The harmonic development of the tide-generating potential. Proc. R. Soc. London Ser. A-Math. Phys. Eng. Sci., 100, 305-329, doi: 10.1098/ rspa.1921.0088. [Link]

Fang, G., Y.-K. Kwok, K. Yu, and Y. Zhu, 1999: Numerical simulation of principal tidal constituents in the South China sea, Gulf of Tonkin and Gulf of Thailand. Cont. Shelf Res., 19, 845-869, doi: 10.1016/s02784343(99)00002-3. [Link]

Farrell, W. E., 1972: Deformation of the Earth by surface loads. Rev. Geophys., 10, 761-797, doi: 10.1029/ rg010i003p00761. [Link]

Hartmann, T. and H. G. Wenzel, 1995: The HW95 tidal potential catalogue. Geophys. Res. Lett., 22, 3553-3556, doi: 10.1029/95g103324. [Link]

Hinderer, J. and D. Crossley, 2004: Scientific achievements from the first phase (1997-2003) of the Global Geodynamics Project using a worldwide network of superconducting gravimeters. J. Geodyn., 38, 237-262, doi: 10.1016/j.jog.2004.07.019. [Link]

Hinderer, J., N. Florsch, J. Mäkinen, H. Legros, and J. E. Faller, 1991: On the calibration of a superconducting gravimeter using absolute gravity measurements. Geophys. J. Int., 106, 491-497, doi: 10.1111/j.1365246x.1991.tb03907.x. [Link]

Hinderer, J., D. Crossley, and R. J. Warburton, 2015: Superconducting Gravimetry. In: Schubert, G. (Ed.), 
Treatise on Geophysics, Second Edition, Volume 3, Elsevier, Oxford, 59-115, doi: 10.1016/B978-0-44453802-4.00062-2. [Link]

Jiang, X. D., D. Y. Li, W. Gong, and C. Y. Bi, 2014: Differential deformation and uplift mechanisms of the eastern and western Tibetan plateau. Chin.J. Geophys., 57, 4016-4028, doi: 10.6038/cjg20141214. (in Chinese) [Link]

Kroner, C. and G. Jentzsch, 1999: Comparison of different barometric pressure reductions for gravity data and resulting consequences. Phys. Earth Planet. Inter., 115, 205-218, doi: 10.1016/s0031-9201(99)00079-5. [Link]

Latychev, K., J. X. Mitrovica, M. Ishii, N. H. Chan, and J. L. Davis, 2009: Body tides on a 3-D elastic earth: Toward a tidal tomography. Earth Planet. Sci. Lett., 277, 86-90, doi: 10.1016/j.eps1.2008.10.008. [Link]

Liang, S., W. Gan, C. Shen, G. Xiao, J. Liu, W. Chen, X. Ding, and D. Zhou, 2013: Three-dimensional velocity field of present-day crustal motion of the Tibetan Plateau derived from GPS measurements. J. Geophys. Res., 118, 5722-5732, doi: 10.1002/2013JB010503. [Link]

Mathews, P. M., 2001: Love numbers and gravimetric factor for diurnal tides. J. Geodetic Soc. Jap., 46, 231-236, doi: 10.11366/sokuchi1954.47.231. [Link]

Matsumoto, K., T. Takanezawa, and M. Ooe, 2000: Ocean tide models developed by assimilating Topex/Poseidon altimeter data into hydrodynamical model: A global model and a regional model around Japan. J. Oceanogr., 56, 567-581, doi: 10.1023/A:1011157212596. [Link]

Melchior, P., 1994: A new data bank for tidal gravity measurements (DB 92). Phys. Earth Planet. Inter., 82, 125155, doi: 10.1016/0031-9201(94)90085-x. [Link]

Melchior, P., B. Ducarme, and O. Francis, 1996: The response of the Earth to tidal body forces described by second- and third-degree spherical harmonics as derived from a 12 year series of measurements with the superconducting gravimeter GWR/T3 in Brussels. Phys. Earth Planet. Inter., 93, 223-238, doi: 10.1016/00319201(95)03073-5. [Link]

Merriam, J. B., 1992: Atmospheric pressure and gravity. Geophys. J. Int., 109, 488-500, doi: 10.1111/j.1365246x.1992.tb00112.x. [Link]

Métivier, L. and C. P. Conrad, 2008: Body tides of a convecting, laterally heterogeneous, and aspherical Earth. J. Geophys. Res., 113, B11405, doi: 10.1029/2007JB005448. [Link]

Meurers, B., 2012: Superconducting gravimeter calibration by CoLocated gravity observations: Results from GWR C025. Int. J. Geophys., 2012, 1-12, doi: 10.1155/2012/954271. [Link]

Riccardi, U., S. Rosat, and J. Hinderer, 2012: On the ac- curacy of the calibration of superconducting gravimeters using absolute and spring sensors: A critical comparison. Pure Appl. Geophys., 169, 1343-1356, doi: 10.1007/s00024-011-0398-8. [Link]

Roosbeek, F., 1996: RATGP95: A harmonic development of the tide-generating potential using an analytical method. Geophys. J. Int., 126, 197-204, doi: 10.1111/j.1365-246X.1996.tb05278.x. [Link]

Schwiderski, E. W., 1980: Ocean tides, part I: Global ocean tidal equations. Mar. Geodesy, 3, 161-217, doi: 10.1080/01490418009387997. [Link]

Shen, Z. K., J. Lü, M. Wang, and R. Bürgmann, 2005: Contemporary crustal deformation around the southeast borderland of the Tibetan Plateau. J. Geophys. Res., 110, B11409, doi: 10.1029/2004JB003421. [Link]

Shin, Y. H., C. K. Shum, C. Braitenberg, S. M. Lee, S.-H. Na, K. S. Choi, H. Hsu, Y.-S. Park, and M. Lim, 2015: Moho topography, ranges and folds of Tibet by analysis of global gravity models and GOCE data. Sci. Rep., 5, doi: 10.1038/srep11681. [Link]

Shukowsky, W. and M. S. M. Mantovani, 1999: Spatial variability of tidal gravity anomalies and its correlation with the effective elastic thickness of the lithosphere. Phys. Earth Planet. Inter., 114, 81-90, doi: 10.1016/ S0031-9201(99)00048-5. [Link]

Sun, H., 1997: Atmospheric gravity Green's functions. Chin. Sci. Bull., 42, 1712-1719, doi: 10.1007/BF02882673. [Link]

Sun, H., H. Xu, B. Ducarme, and J. Hinderer, 1999: Comprehensive comparison and analysis of the tidal gravity observations obtained with superconducting gravimeters at stations in China, Belgium and France. Chin. Sci. Bull., 44, 750-755, doi: 10.1007/BF02909719. [Link]

Sun, H., X. Chen, H. Hsu, and Y. Wang, 2001: Accurate determination of calibration factor for tidal gravity observation of a GWR-superconducting gravimeter. Acta Seismol. Sin., 14, 692-700, doi: 10.1007/bf02718080. [Link]

Sun, H., J. Xu, and B. Ducarme, 2003: Experimental earth tidal models in considering nearly diurnal free wobble of the Earth's liquid core. Chin. Sci. Bull., 48, 935-940, doi: 10.1007/BF03325679. [Link]

Sun, H., B. Ducarme, H. Xu, L. Vandercoilden, J. Xu, and J. Zhou, 2005a: Adaptability of the ocean and earth tidal models based on global observations of the superconducting gravimeters. Sci. China Ser. D, 48, 1859-1869, doi: 10.1360/04yd0071. [Link]

Sun, H., H. Hsu, J. C. Zhou, X. D. Chen, J. Q. Xu, B. L. Zhou, X. H. Hao, and M. Liu, 2005b: Latest observational results from the superconducting gravimeter at station Wuhan and investigation of ocean tide models. Chin. J. Geophys., 48, 331-341, doi: 10.1002/cjg2.658. [Link] 
Sun, H., J. Xu, X. Chen, J. Zhou, and X. Le, 2013: Results of Gravity Observations Using a Superconducting Gravimeter at the Tibetan Plateau. Terr. Atmos. Ocean. Sci., 24,541-550, doi: 10.3319/TAO.2012.10.25.02(TibXS). [Link]

Teng, J. W., X. M. Ruan, Y. Q. Zhang, G. Z. Hu, and Y. F. Yang, 2012: The stratificational velocity structure of crust and covering strata of upper mantle and the orbit of deep interaquifer substance locus of movement for Tibetan Plateau. Acta Petrol. Sin., 28, 4077-4100.

Van Camp, M., B. Meurers, O. De Viron, and T. Forbriger, 2016: Optimized strategy for the calibration of superconducting gravimeters at the one per mille level. $J$. Geod., 90, 91-99, doi: 10.1007/s00190-015-0856-7. [Link]

Vauterin, P., 1998: Tsoft: Graphical \& interactive software for the analysis of Earth tide data. In: Ducarme, B. and P. Paquet (Eds.), Proceedings of the 13th International Symposium on the Earth Tides, Brussels, 481-486.

Wahr, J. M., 1981: Body tides on an elliptical, rotating, elastic and oceanless earth. Geophys. J. R. Astr. Soc., 64, 677-703, doi: 10.1111/j.1365-246X.1981.tb02690.x. [Link]

Wang, Q., P.-Z. Zhang, J. T. Freymueller, R. Bilham, K. M. Larson, X. Lai, X. You, Z. Niu, J. Wu, Y. Li, J. Liu, Z. Yang, and Q. Chen, 2001: Present-day crustal deformation in China constrained by global positioning system measurements. Science, 294, 574-577, doi: 10.1126/science.1063647. [Link]

Wei, J., H. Li, Z. W. Liu, K. X. Kang, and H. T. Hao, 2012: Observation of superconducting gravimeter at Jiufeng seismic station. Chin. J. Geophys., 55, 1894-1902, doi: 10.6038/j.issn.0001-5733.2012.06.010. [Link]

Wenzel, H. G., 1996: The nanogal software: Earth tide data processing package ETERNA 3.30. Bull. Inf. Marées
Terr., 124, 9425-9439.

Xu, C., Z. Liu, Z. Luo, Y. Wu, and H. Wang, 2017: Moho topography of the Tibetan Plateau using multi-scale gravity analysis and its tectonic implications. $J$. Asian Earth Sci., 138, 378-386, doi: 10.1016/j.jseaes.2017.02.028. [Link]

Xu, H., H. Sun, J. Xu, and G. Tao, 2000: International tidal gravity reference values at Wuhan station. Sci. China Ser. $D, 43,77-83$, doi: 10.1007/bf02877832. [Link]

$\mathrm{Xu}, \mathrm{J}$. and H. Sun, 2003: Deformation response of a SNREI Earth to surface loads and tidal forces. Chin. J. Geophys., 46, 465-477, doi: 10.1002/cjg2.3364. (in Chinese) [Link]

Xu, J., H. Sun, and B. Ducarme, 2004: A Global Experimental Model for Gravity Tides of the Earth. J. Geodyn., 38, 293-306, doi: 10.1016/j.jog.2004.07.003. [Link]

Xu, J., X. Chen, J. Zhou, and H. Sun, 2012: Characteristics of tidal gravity changes in Lhasa, Tibet, China. Chin. Sci. Bull., 57, 2586-2594, doi: 10.1007/s11434-0125130-2. [Link]

Xu, J., J. C. Zhou, X. D. Chen, and H. P. Sun, 2014: Longterm Observations of Gravity Tides from a Superconducting Gravimeter at Wuhan. Chin. J. Geophys., 57, 3091-3102, doi: 10.6038/cjg20141001. (in Chinese) [Link]

Yao, H., R. D. van der Hilst, and J. P. Montagner, 2010: Heterogeneity and anisotropy of the lithosphere of SE Tibet from surface wave array tomography. J. Geophys. Res., 115, B12307, doi: 10.1029/2009jb007142. [Link]

Zhang, Z., Y. Deng, J. Teng, C. Wang, R. Gao, Y. Chen, and W. Fan, 2011: An overview of the crustal structure of the Tibetan plateau after 35 years of deep seismic soundings. J. Asian Earth Sci., 40, 977-989, doi: 10.1016/j.jseaes.2010.03.010. [Link] 